\title{
Management of bleeding in patients treated with direct oral anticoagulants
}

Marcel Levi ${ }^{1,2}$

\begin{abstract}
Background: Recently, a new generation of direct-acting oral anticoagulants (DOACs) with a greater specificity towards activated coagulation factors was introduced based on encouraging results for efficacy and safety in clinical studies. An initial limitation of these new drugs was the absence of an adequate strategy to reverse the effect if a bleeding event occurs or an urgent invasive procedure has to be carried out.

Main text: Specific reversing agents for DOACs have become available, however, and are now evaluated in clinical studies. For the anti-factor Xa agents (rivaroxaban, apixaban, and edoxaban) a number of studies have shown that the administration of prothrombin complex concentrate resulted in a correction of the prolonged prothrombin time and restored depressed thrombin generation after rivaroxaban treatment in a controlled trial in healthy human subjects. In view of the relatively wide availability of prothrombin complex concentrates, this would be an interesting option if the results can be confirmed in patients on oral factor Xa inhibitors who present with bleeding complications. More specific reversal can be achieved with andexanet, a new agent currently in development that competitively binds to the anti-factor Xa agents. For the direct thrombin inhibitor dabigatran, the administration of prothrombin complex concentrates showed variable results in various volunteer trials and efficacy at relatively high doses in animal studies. Recently, a Fab fragment of a monoclonal antibody (idarucizumab) was shown to be an effective reversal agent for dabigatran in human studies.
\end{abstract}

Conclusion: For the new generation of DOACs, several reversal strategies and specific antidotes are under evaluation, although most interventions need further evaluation in clinical trials.

Keywords: Anticoagulants, Hemorrhage, Direct-acting oral anticoagulants, Dabigatran, Rivaroxaban, Apixaban, Edoxaban

\section{Background}

Anticoagulants are frequently prescribed agents for the prevention and treatment of a myriad of cardiovascular conditions. Conventional anticoagulant agents, such as vitamin K antagonists (VKAs; warfarin, phenprocoumon, or coumadin) and heparin or low molecular weight (LMW) heparin, are increasingly replaced by direct oral anticoagulants (DOACs) directly inhibiting factor Xa (e.g., rivaroxaban, apixaban, or edoxaban) or factor IIa (e.g., dabigatran). This new generation of anticoagulants is termed novel oral anticoagulants (NOACs) or DOACs. A large number of clinical studies have shown that these

\footnotetext{
Correspondence: m.m.levi@amc.uva.nl
${ }^{1}$ Department of Vascular Medicine, Academic Medical Center (E-2), University

Correspondence: m.m.levi@amc.uva.nl
${ }^{1}$ Department of Vascular Medicine, Academic Medical Center (E-2), University of Amsterdam, Meibergdreef 9, 1105 AZ Amsterdam, The Netherlands ${ }^{2}$ Department of Medicine, University of Amsterdam, Amsterdam, The Netherlands
}

(c) 2016 The Author(s). Open Access This article is distributed under the terms of the Creative Commons Attribution 4.0 International License (http://creativecommons.org/licenses/by/4.0/), which permits unrestricted use, distribution, and reproduction in any medium, provided you give appropriate credit to the original author(s) and the source, provide a link to the Creative Commons license, and indicate if changes were made. The Creative Commons Public Domain Dedication waiver (http://creativecommons.org/publicdomain/zero/1.0/) applies to the data made available in this article, unless otherwise stated. embolic diseases [1].

The most important complication of treatment with anticoagulant agents is hemorrhage, which may be serious, may cause long-term debilitating disease, or may even be life-threatening [2]. Bleeding in a patient on anticoagulants may require specific (additional) management; if the bleeding situation is sufficiently severe, swift reversal of the anticoagulant effect of the anticoagulant agent may even be required. Depending on the clinical situation, including the site and/or the severity of the bleeding, this reversal may take place in a few hours, but in some cases immediate reversal is needed [3]. Generally, each (immediate) reversal of anticoagulant treatment needs also to take into consideration the indication for the antithrombotic agents. For example, the immediate interruption of anticoagulants in a patient with recent venous thromboembolism will markedly increase the 
short-term risk of recurrent venous thrombosis or pulmonary embolism. Likewise, in a patient with advanced heart disease and atrial fibrillation, interruption of anticoagulants may increase the risk of cerebral or systemic embolism. Each of these specific clinical settings requires a careful and balanced individual assessment of the benefits and dangers of reversing anticoagulants (and potential strategies to keep the period of reversal as brief as possible). Here we will briefly describe the epidemiology of bleeding complications due to anticoagulants and various strategies to reverse the anticoagulant effect of antithrombotic agents, focusing on the new generation of anticoagulants (DOACs).

\section{Relevance, incidence, and risk factors for bleeding in patients on anticoagulant treatment}

The relevance of hemorrhagic complications in patients on anticoagulants is clearly demonstrated in a series of observational studies. In a large study of 34,146 patients with acute ischemic coronary syndromes, anticoagulantassociated bleeding was associated with a 5 -fold increased risk of death during the first month and a 1.5-fold higher mortality between 30 days and 6 months [4]. Major hemorrhage was an independent predictor of mortality across all subgroups that were analyzed.

Currently, VKAs are still the most frequently used oral anticoagulant agents for long-term prevention and treatment of a wide range of cardiovascular diseases. In wellcontrolled patients in clinical trials, treatment with VKAs increases the risk of major bleeding by $0.5-1.0 \%$ /year and increases the risk of intracranial hemorrhage by about 0.2-0.3\%/year [5]. However, in three real-life surveys this incidence varied from 1.4 to $3.4 \%$ year [6]. One needs to realize that the relatively uncomplicated trial populations may poorly reflect the real-life setting in which anticoagulants are prescribed. For example, in six pivotal trials that demonstrated the superiority of warfarin over placebo in the prevention of thromboembolic complications in patients with atrial fibrillation, 28,787 patients were screened but only $12.6 \%$ of these patients were included in the studies [7].

The incidence of hemorrhagic complications with use of the new generation of oral anticoagulants with direct inhibitory properties towards thrombin or factor $\mathrm{Xa}$ is not very different. The severity and incidence of bleeding events in DOAC-treated patients has been evaluated in a number of clinical studies, which demonstrated similar or lower rates of major bleeding with DOACs compared with traditional anticoagulants [8-11]. The safety and efficacy of DOACs versus warfarin were evaluated in a meta-analysis of 14 prospective and retrospective studies in AF patients undergoing catheter ablation $(4782$ patients in total) [12]. Overall, no significant difference was observed in terms of thromboembolic events or major bleeding events between the warfarin and dabigatran treatment arms, although minor bleeding events occurred significantly less frequently with dabigatran compared with warfarin. Of note, DOAC treatment was associated with a reduced intracranial hemorrhage incidence compared with warfarin therapy, whereas some analyses showed an increased risk for gastrointestinal bleeds [12-14]. The recent guidelines from the European Society of Cardiology on anticoagulant management of atrial fibrillation recommend non-VKAs based on their more favorable incidence of hemorrhagic complications [15]. Likewise, DOACs are also advocated for the treatment of venous thromboembolism in recent guidelines [16]. Although these clinical study results are encouraging, there is very limited real-world evidence with regards to the incidence and severity of DOAC-related bleeding events; most of the currently available data are based on postmarketing studies.

Some clinical studies have reported on their experience with bleeding complications in patients treated with DOACs compared with patients on warfarin $[17,18]$. Data from the EINSTEIN studies on rivaroxaban demonstrated that patients who presented with major bleeding on rivaroxaban had a relatively milder presentation and a better recovery compared with patients on warfarin. A prospective chart review of 15 emergency department patients treated with dabigatran and 123 patients treated with warfarin showed that patients on dabigatran had a shorter length of stay, fewer major bleeding events, and fewer life-threatening complications compared with patients on warfarin. Additionally, dabigatran use was more commonly associated with gastrointestinal hemorrhage, and less frequently associated with intracranial bleeding, compared with warfarin, in this patient population. This finding is in contrast to results from an analysis of insurance claim and administrative data from the FDA Mini-Sentinel database, which showed that dabigatran was associated with reduced incidence of gastrointestinal bleeds compared with warfarin [19]. The Australian Therapeutic Goods Administration has been monitoring the safety profile of dabigatran, and reported 361 serious bleeding events with this agent between 2011 and 2013 [20]. The most commonly reported bleeding site was the gastrointestinal tract, while a smaller proportion of intracranial bleeds were reported for dabigatran versus warfarin [20].

The most important risk factor for hemorrhage in users of anticoagulants is the intensity of the anticoagulant dose [21]. Studies suggest that with a target international normalized ratio (INR) of $>3.0$ the incidence of major bleeding is twice as large as in studies with a target INR of 2.0-3.0 [22]. In a meta-analysis of studies in patients with prosthetic heart valves, a lower INR target range resulted in a lower frequency of major 
bleeding and intracranial hemorrhage with a similar antithrombotic efficacy [23]. For dabigatran a clear relationship between dose and incidence of bleeding complications has been demonstrated in a clinical study in patients with atrial fibrillation [8], and all other DOACs have also demonstrated a similar dose-adverse event relationship [14].

Patient characteristics constitute another important determinant of the bleeding risk. Older patients have a 2fold increased risk of bleeding [24], and the relative risk of intracranial hemorrhage (in particular at higher intensities of anticoagulation) was 2.5 (95\% CI 2.3-9.4) in patients aged $>85$ years compared with patients $70-74$ years old [25]. Comorbidity may also significantly increase the risk of bleeding. A case-control study in 1986 patients on anticoagulants showed that comorbidity (such as mild renal insufficiency, hepatic dysfunction, or diabetes) increased the risk of bleeding by about 2.5 [7]. Another very important determinant of the risk of bleeding is the combined use of medication that affects both the coagulation system and platelet function. Two meta-analyses, comprising six trials with a total of 3874 patients and 10 trials with a total of 5938 patients, found a relative risk of major bleeding when antithrombotic agents were combined with aspirin of 2.4 (95\% CI 1.2-4.8) and 2.5 (95\% CI 1.7-3.7), respectively [26]. A population-based case-control study confirmed the high risk of upper gastrointestinal bleeding in patients using anticoagulants in combination with aspirin and/or clopidogrel [27]. It should be noted that the combined use of the new anticoagulant agents and antiplatelet agents was discouraged in the clinical trials, whereas this is increasingly common in clinical practice and may also have a serious impact on the risk of hemorrhage. Nonsteroidal anti-inflammatory agents (NSAIDs) are also associated with an enhanced risk of gastrointestinal bleeding. The combined use of anticoagulants and NSAIDs may result in an 11-fold higher risk of hospitalization for gastrointestinal bleeding as compared with the general population [28]. This risk is not significantly lower when using selective inhibitors of COX-2. As DOACs are associated with a higher risk of gastrointestinal bleeding compared with conventional anticoagulants (see above), the effect of combined treatment with these agents and aspirin or NSAIDs may be even larger.

\section{Reversal of DOACs}

One of the main advantages of DOACs are the relatively stable pharmacokinetic and pharmacodynamic properties, which obviates the need for repeated control of the intensity of anticoagulation and dose adjustments. This means that for some clinical situations these drugs may represent an important improvement, but as already indicated the risk of (major) bleeding is still present. Clinical trials in patients using DOACs agents excluded many patients with common comorbidities and discouraged the simultaneous use of agents affecting platelet function. The risk of hemorrhage in these trials may therefore represent an underestimation of real-life bleeding risk.

Dependent on the severity of the clinical situation and in view of the relatively short half-life of the direct factor Xa inhibitors (5-15 h), cessation of medication may often be sufficient to reverse the anticoagulant effect in case of bleeding. Some authors argue that in most cases this will suffice and more immediate reversal is hardly ever really needed in clinical practice [29]. However, if immediate reversal of anticoagulation is deemed necessary, additional measures may be required. In general, these can be divided into nonspecific and specific interventions to reverse the anticoagulant effect (Table 1). A practical approach to the patient presenting with bleeding while using DOACs, based on recommendations of the European Heart Rhythm Association [15], is presented in Table 2.

Nonspecific measures include (activated) prothrombin complex concentrates (PCCs) or recombinant factor VIIa (rFVIIa). The prothrombotic potential of activated PCCs and rFVIIa might be higher than that of nonactivated PCCs, so nonactivated PCCs may be preferred [30, 31]. In addition, a recent retrospective series of bleeding patients treated with PCCs for anticoagulant reversal showed a $20 \%$ risk of thromboembolic complications, although part of the risk may have been due to the underlying thromboembolic risk for which the anticoagulant was prescribed in the first place and the clinical situation of the patients [32].

Specific measures are directly targeting the anticoagulant agent, by means of (Fab fragments of) monoclonal antibodies (in the case of dabigatran) or molecules that competitively bind to the anticoagulant agents (in the case of factor Xa inhibitors).

Table 1 Pharmacological options for reversing the effect of the direct-acting oral anticoagulants

\begin{tabular}{ll}
\hline Inhibitor & Conventional dose \\
\hline Nonspecific reversal (prohemostatic interventions) & \\
Prothrombin complex concentrates & $50 \mathrm{U} / \mathrm{kg}$ \\
Activated prothrombin complex concentrates & $50 \mathrm{U} / \mathrm{kg}$ \\
Recombinant factor Vlla & $90 \mu \mathrm{g} / \mathrm{kg}$ \\
Specific reversal & \\
Directed at dabigatran & \\
Idarucizumab & $5 \mathrm{~g}$ \\
Directed at rivaroxaban, apixaban, and edoxaban & \\
Andexanet-alfa & $600-800 \mathrm{mg}$ \\
Ciraparantag & $100 \mathrm{mg}$ \\
\hline
\end{tabular}


Table 2 Practical guide for how to manage bleeding complications in patients on direct oral anticoagulants

\begin{tabular}{l}
$\begin{array}{l}\text { Oral thrombin inhibitors } \\
\text { (dabigatran) }\end{array}$ \\
\hline None life-threatening bleeding \\
$\begin{array}{l}\text { Check last intake; restoration of normal coagulation Xa inhibitors } \\
\text { (rivaroxaban, apixaban, edoxaban) }\end{array}$ \\
to be expected at $12-24 \mathrm{~h}$ (in case of creatinin clearance \\
$>80 \mathrm{ml} / \mathrm{min}$ ) or $24-36 \mathrm{~h}$ (in case of creatinin clearance \\
$50-80 \mathrm{ml} / \mathrm{min}$ ) \\
Local hemostatic interventions, fluid management, transfusion \\
Consider tranexamic acid (1000 $\mathrm{mg} 3 \mathrm{dd}$ ) or DDAVP $(0.3 \mu \mathrm{g} / \mathrm{kg})$
\end{tabular}

Life-threatening bleeding

$\begin{array}{ll}\text { All of the above } & \text { All of the above } \\ \text { Idarucizumab } & \begin{array}{l}\text { Andexanet alfa } \\ \text { Ciraparantag (under investigation) }\end{array} \\ \begin{array}{ll}\text { Prothrombin complex concentrate } \\ \text { (no evidence) }\end{array} & \begin{array}{l}\text { Prothrombin complex concentrate } \\ \text { (healthy volunteer data) }\end{array} \\ \text { Activated PCC (no evidence) } & \text { Activated PCC (no human evidence) } \\ \text { Recombinant factor Vlla } & \text { Recombinant factor Vlla } \\ \text { (no evidence) } & \text { (healthy volunteer data) }\end{array}$

Suggested management strategy in case of hemorrhagic complications in patients using direct oral anticoagulants, modified from [15] $P C C$ prothrombin complex concentrate, $d d$ daily, DDAVP de-amino D-arginine vasopressin

\section{Reversal of direct oral factor Xa inhibitors}

Preclinical data suggest that rFVIIa and PCCs (activated and nonactivated) may be useful for the reversal of NOAC-induced anticoagulation. Experimental studies have demonstrated that the amelioration of coagulation parameters is associated with a beneficial effect on blood loss [33, 34]. In addition, a number of studies in human healthy subjects have revealed that the administration of PCC resulted in a correction of the prolonged prothrombin time and restored depressed thrombin generation after rivaroxaban treatment in a controlled trial in healthy human subjects. Similarly, a three-factor PCC (Profilnine ${ }^{\circledast}$; Grifols Biologicals Inc., Los Angeles, CA, USA) was also evaluated for rivaroxaban reversal in a study in healthy volunteers and was shown capable of correcting some of the rivaroxaban-induced effects on coagulation parameters [35-37]. Recent studies confirmed these findings also at lower doses of PCCs [38, 39].

More specific reversal of anti-factor Xa agents can be achieved with new agents that competitively bind to the anti-factor Xa agents. Ciraparantag binds directly to the factor Xa agent (in particular edoxaban) via hydrogen bonds from or to various parts of the molecule [40, 41]. This antidote was shown to block the anticoagulant effect of edoxaban and restored the prothrombin time in vitro. Further development is ongoing. Similarly, andexanet-alfa is a recombinant protein analog of factor Xa that binds to factor Xa inhibitors but does not trigger prothrombotic activity. Andexanet virtually immediately reversed the anticoagulant activity of apixaban and rivaroxaban in healthy subjects without evidence of clinical toxic effects [42]. A clinical study in patients who present with bleeding while taking anti-factor Xa DOACs is ongoing.

Monitoring the reversal of the anticoagulant effect of factor Xa inhibitors is most simply done by measuring the prothrombin time, although there is some variability between prothrombin time reagents and for some agents the anti-factor Xa assay is more reliable [43]. Of note, the INR is not a suitable test to quantitate the (residual) anticoagulant effect by factor Xa agents.

\section{Reversal of direct oral thrombin inhibitors}

The other group of DOACs directly targets thrombin (factor IIa) and is represented by dabigatran. Preclinical studies show variable effects for the efficacy of (activated) PCCs and factor VIIa to reverse the anticoagulant effect and to ameliorate experimental bleeding in animals exposed to dabigatran [33, 44, 45] Relatively high doses of PCCs, however, seem to have a reversing effect. Similarly, human volunteer studies show a limited effect of conventional doses of PCC to normalize coagulation parameters after ingestion of dabigatran [35, 37]. There are no systematic clinical trials investigating the effect of PCCs to reverse dabigatran-associated hemorrhage in clinical practice. However, some case series are helpful. The effectiveness of dabigatran-related bleeding management using a nonactivated four-factor (4F)-PCC (Octaplex; Octapharma, Vienna, Austria) was recently evaluated by Diaz et al. [46]. Five patients receiving dabigatran, 76-88 years of age, were administered 4 F-PCC to manage dabigatran-related GI bleeding complications. Treatment with 4 F-PCC was able to adequately control bleeding in four of five patients; the fifth patient died of septic shock and coagulopathy secondary to severe hemorrhage. No thromboembolic events were reported in the next 6 months of follow-up in these patients. Interestingly, the authors also reported that, in the one patient presenting with a prolonged activated partial thromboplastin time (aPTT) as a result of dabigatran treatment, administration of 4 F-PCC was able to partially normalize this laboratory parameter. Similar clinical case reports have been reviewed elsewhere [47].

A direct reversing agent for dabigatran has been developed recently and is based on a Fab fragment of a monoclonal antibody (idaricuzimab) directly binding to dabigatran and eliminating its anticoagulant effect [48]. Experimental studies show a rapid and almost immediate reversal of dabigatran-induced anticoagulation $[49,50]$. In healthy human subjects that were exposed to dabigatran, this antidote also showed a complete elimination of the anticoagulant effect of dabigatran [51]. In a clinical trial in 90 patients on dabigatran who had serious bleeding or required an urgent invasive procedure, idarucizumab 
completely reversed the anticoagulant effect of dabigatran almost instantaneously [52].

Monitoring of the anticoagulant effect of thrombin inhibitors in routine clinical practice is difficult. The aPTT is not very useful. An ecarin clotting time may be more accurate but is not readily available in most routine clinical settings. Most convenient and practically applicable for monitoring of the anticoagulant effect may be the diluted thrombin time, which needs to be standardized for the specific agent that was used [53].

\section{Conclusion}

For the new generation of DOACs, several reversal strategies are under evaluation and specific antidotes or reversing agents are being developed, although most interventions need further evaluation in clinical trials. Such strategies can be used in case of serious hemorrhage complicating the use of DOACs or if a patient on DOACs needs to undergo an immediate invasive procedure.

\section{Abbreviations}

aPTT, activated partial thromboplastin time; DOAC, direct oral anticoagulant; 4F, four-factor; INR, international normalized ratio; NOAC, novel oral anticoagulant; NSAID, nonsteroidal anti-inflammatory agent; PCC, prothrombin complex concentrate; RFVIIa, recombinant factor VIla; VKA, vitamin $\mathrm{K}$ antagonist

\section{Authors' contributions}

ML designed and undertook the search strategies, screened search results, organized retrieval of papers, appraised the quality of and extracted data from papers, drafted the manuscript, and wrote the review. The author has read and approved the final version of the manuscript.

\section{Competing interests}

$\mathrm{ML}$ has been principle investigator in the execution of clinical trials with PCCs to reverse anticoagulants sponsored by Sanquin (the Netherlands) and Johnson \& Johnson (USA). There are no further competing interests to declare.

\section{Published online: 20 August 2016}

\section{References}

1. Hirsh J, Guyatt G, Albers GW, Harrington R, Schunemann HJ. Antithrombotic and thrombolytic therapy: American College of Chest Physicians EvidenceBased Clinical Practice Guidelines (8th Edition). Chest. 2008;133(6 Suppl): 110S-2S.

2. Mannucci PM, Levi M. Prevention and treatment of major blood loss. N Engl J Med. 2007;356(22):2301-11.

3. Levi MM, Eerenberg E, Lowenberg E, Kamphuisen PW. Bleeding in patients using new anticoagulants or antiplatelet agents: risk factors and management. Neth J Med. 2010;68(2):68-76.

4. Eikelboom JW, Mehta SR, Anand SS, Xie C, Fox KA, Yusuf S. Adverse impact of bleeding on prognosis in patients with acute coronary syndromes. Circulation. 2006;114(8):774-82.

5. Ageno W, Gallus AS, Wittkowsky A, Crowther M, Hylek EM, Palareti G. Oral anticoagulant therapy: antithrombotic therapy and prevention of thrombosis, 9th ed: American College of Chest Physicians Evidence-Based Clinical Practice Guidelines. Chest. 2012;141(2 Suppl):e44S-88S.

6. Levi M, Eerenberg E, Kamphuisen PW. Bleeding risk and reversal strategies for old and new anticoagulants and anti-platelet agents. J Thromb Haemost. 2011;9:1705-12.

7. Levi M, Hovingh GK, Cannegieter SC, Vermeulen M, Buller HR, Rosendaal FR. Bleeding in patients receiving vitamin $\mathrm{K}$ antagonists who would have been excluded from trials on which the indication for anticoagulation was based. Blood. 2008;111(9):4471-6.
8. Connolly SJ, Ezekowitz MD, Yusuf S, Eikelboom J, Oldgren J, Parekh A, et al. Dabigatran versus warfarin in patients with atrial fibrillation. N Engl J Med. 2009;361(12):1139-51.

9. Patel MR, Mahaffey KW, Garg J, Pan G, Singer DE, Hacke W, et al. Rivaroxaban versus warfarin in nonvalvular atrial fibrillation. N Engl J Med. 2011;365(10):883-91.

10. Schulman S, Kearon C, Kakkar AK, Mismetti P, Schellong S, Eriksson H, et al. Dabigatran versus warfarin in the treatment of acute venous thromboembolism. N Engl J Med. 2009;361(24):2342-52.

11. Buller HR, Prins MH, Lensin AW, Decousus $H$, Jacobson BF, Minar E, et al. Oral rivaroxaban for the treatment of symptomatic pulmonary embolism. N Engl J Med. 2012;366(14):1287-97.

12. Providencia R, Albenque JP, Combes S, Bouzeman A, Casteigt B, Combes N, et al. Safety and efficacy of dabigatran versus warfarin in patients undergoing catheter ablation of atrial fibrillation: a systematic review and meta-analysis. Heart. 2014;100(4):324-35.

13. Desai J, Granger CB, Weitz Jl, Aisenberg J. Novel oral anticoagulants in gastroenterology practice. Gastrointest Endosc. 2013;78(2):227-39.

14. van Es N, Coppens M, Schulman S, Middeldorp S, Buller HR. Direct oral anticoagulants compared with vitamin $\mathrm{K}$ antagonists for acute symptomatic venous thromboembolism: evidence from phase 3 trials. Blood. 2014; 124(12):1968-75.

15. Heidbuchel H, Verhamme P, Alings M, Antz M, Diener HC, Hacke W, et al. Updated European Heart Rhythm Association practical guide on the use of non-vitamin-K antagonist anticoagulants in patients with non-valvular atrial fibrillation: Executive summary-Revision 1. Eur Heart J. 2016. Epub ahead of print.

16. Kearon C, Akl EA, Comerota AJ, Prandoni P, Bounameaux H, Goldhaber SZ, et al. Antithrombotic therapy for vte disease: antithrombotic therapy and prevention of thrombosis, 9th ed: American College of Chest Physicians Evidence-Based Clinical Practice Guidelines. Chest. 2012;141(2 Suppl): e419S-94S.

17. Berger R, Salhanick SD, Chase M, Ganetsky M. Hemorrhagic complications in emergency department patients who are receiving dabigatran compared with warfarin. Ann Emerg Med. 2013;61(4):475-9.

18. Eerenberg ES, Middeldorp S, Levi M, Lensing AW, Buller HR. Clinical impact and course of major bleeding with rivaroxaban and vitamin $\mathrm{K}$ antagonists. J Thromb Haemost. 2015;13(9):1590-6.

19. Southworth MR, Reichman ME, Unger EF. Dabigatran and postmarketing reports of bleeding. N Engl J Med. 2013;368(14):1272-4.

20. Chen EY, Diug B, Bell JS, Mc Namara KP, Dooley MJ, Kirkpatrick CM, et al. Spontaneously reported haemorrhagic adverse events associated with rivaroxaban and dabigatran in Australia. Ther Adv Drug Saf. 2016;7(1):4-10.

21. Schulman S, Beyth RJ, Kearon C, Levine MN. Hemorrhagic complications of anticoagulant and thrombolytic treatment: American College of Chest Physicians Evidence-Based Clinical Practice Guidelines (8th Edition). Chest. 2008;133(6 Suppl):257S-98S.

22. Saour JN, Sieck JO, Mamo LA, Gallus AS. Trial of different intensities of anticoagulation in patients with prosthetic heart valves. N Engl J Med. 1990;322(7):428-32.

23. Vink R, Kraaijenhagen RA, Hutten BA, van den Brink RB, de Mol BA, Buller $\mathrm{HR}$, et al. The optimal intensity of vitamin $\mathrm{K}$ antagonists in patients with mechanical heart valves: a meta-analysis. J Am Coll Cardiol. 2003; 42(12):2042-8.

24. Hutten BA, Lensing AW, Kraaijenhagen RA, Prins MH. Safety of treatment with oral anticoagulants in the elderly. A systematic review. Drugs Aging. 1999;14(4):303-12.

25. Fang MC, Chang Y, Hylek EM, Rosand J, Greenberg SM, Go AS, et al. Advanced age, anticoagulation intensity, and risk for intracranial hemorrhage among patients taking warfarin for atrial fibrillation. Ann Intern Med. 2004; 141(10):745-52.

26. Rothberg MB, Celestin C, Fiore LD, Lawler E, Cook JR. Warfarin plus aspirin after myocardial infarction or the acute coronary syndrome: meta-analysis with estimates of risk and benefit. Ann Intern Med. 2005;143(4):241-50.

27. Hallas J, Dall M, Andries A, Andersen BS, Aalykke C, Hansen JM, et al. Use of single and combined antithrombotic therapy and risk of serious upper gastrointestinal bleeding: population based case-control study. BMJ. 2006; 333(7571):726

28. Mellemkjaer L, Blot WJ, Sorensen HT, Thomassen L, McLaughlin JK, Nielsen $\mathrm{GL}$, et al. Upper gastrointestinal bleeding among users of NSAIDs: 
a population-based cohort study in Denmark. Br J Clin Pharmacol. 2002; 53(2):173-81.

29. Eerenberg ES, Levi M, Buller HR. Contra: "Antidotes for novel anticoagulants?" - Do we really need them. Thromb Haemost. 2012;108(4):623-4.

30. Miesbach W, Seifried E. New direct oral anticoagulants-current therapeutic options and treatment recommendations for bleeding complications. Thromb Haemost. 2012;108(4):625-32.

31. Levi M, Levy JH, Andersen HF, Truloff D. Safety of recombinant activated factor VII in randomized clinical trials. N Engl J Med. 2010; 363(19):1791-800

32. Sridharan M, Wysokinski WE, Pruthi R, Oyen L, Freeman WD, Rabinstein AA, et al. Periprocedural warfarin reversal with prothrombin complex concentrate. Thromb Res. 2016;139:160-5.

33. Lambourne MD, Eltringham-Smith $\sqcup$, Gataiance $S$, Arnold DM, Crowther MA, Sheffield WP. Prothrombin complex concentrates reduce blood loss in murine coagulopathy induced by warfarin, but not in that induced by dabigatran etexilate. J Thromb Haemost. 2012;10(9):1830-40.

34. Fukuda T, Honda Y, Kamisato C, Morishima Y, Shibano T. Reversal of anticoagulant effects of edoxaban, an oral, direct factor Xa inhibitor, with haemostatic agents. Thromb Haemost. 2012;107(2):253-9.

35. Eerenberg ES, Kamphuisen PW, Sijpkens MK, Meijers JC, Buller HR, Levi M. Reversal of rivaroxaban and dabigatran by prothrombin complex concentrate: a randomized, placebo-controlled, crossover study in healthy subjects. Circulation. 2011;124(14):1573-9.

36. Levi M, Moore KT, Castillejos CF, Kubitza D, Berkowitz SD, Goldhaber SZ, et al. Comparison of three-factor and four-factor prothrombin complex concentrates regarding reversal of the anticoagulant effects of rivaroxaban in healthy volunteers. J Thromb Haemost. 2014;12(9):1428-36.

37. Marlu R, Hodaj E, Paris A, Albaladejo P, Cracowski JL, Pernod G. Effect of non-specific reversal agents on anticoagulant activity of dabigatran and rivaroxaban: a randomised crossover ex-vivo study in healthy volunteers. Thromb Haemost. 2012;108:217-24.

38. Cheung YW, Barco S, Hutten BA, Meijers JC, Middeldorp S, Coppens M. In vivo increase in thrombin generation by four-factor prothrombin complex concentrate in apixaban-treated healthy volunteers. J Thromb Haemost. 2015;13(10):1799-805.

39. Barco S, Whitney Cheung Y, Coppens M, Hutten BA, Meijers JC, Middeldorp S. In vivo reversal of the anticoagulant effect of rivaroxaban with four-factor prothrombin complex concentrate. Br J Haematol. 2016; 172(2):255-61.

40. Ansell JE, Bakhru SH, Laulicht BE, Steiner SS, Grosso M, Brown K, et al. Use of PER977 to reverse the anticoagulant effect of edoxaban. N Engl J Med. 2014;371(22):2141-2.

41. Ansell J. Blocking bleeding: reversing anticoagulant therapy. Nat Med. 2013; 19(4):402-4.

42. Siegal DM, Curnutte JT, Connolly SJ, Lu G, Conley PB, Wiens BL, et al. Andexanet alfa for the reversal of factor Xa inhibitor activity. N Engl J Med. 2015;373(25):2413-24.

43. Hillarp A, Baghaei F, Fagerberg Bl, Gustafsson KM, Stigendal L, Sten-Linder M, et al. Effects of the oral, direct factor Xa inhibitor rivaroxaban on commonly used coagulation assays. J Thromb Haemost. 2011;9(1):133-9.

44. Zhou W, Zorn M, Nawroth P, Butehorn U, Perzborn E, Heitmeier S, et al. Hemostatic therapy in experimental intracerebral hemorrhage associated with rivaroxaban. Stroke. 2013;44(3):771-8

45. Honickel M, Maron B, van Ryn J, Braunschweig T, Ten Cate H, Spronk HM, et al. Therapy with activated prothrombin complex concentrate is effective in reducing dabigatran-associated blood loss in a porcine polytrauma model. Thromb Haemost. 2016;1 15(2):271-84.

46. Diaz MQ, Borobia AM, Nunez MA, Virto AM, Fabra S, Casado MS, et al. Use of prothrombin complex concentrates for urgent reversal of dabigatran in the emergency department. Haematologica. 2013;98(11):e143-4.

47. Levy JH, Levi M. New oral anticoagulant-induced bleeding: clinical presentation and management. Clin Lab Med. 2014;34(3):575-86.

48. Eikelboom JW, Quinlan DJ, van Ryn J, Weitz Jl. Idarucizumab: the antidote for reversal of dabigatran. Circulation. 2015;132(25):2412-22.

49. Grottke O, Honickel M, van Ryn J, ten Cate H, Rossaint R, Spronk HM. Idarucizumab, a specific dabigatran reversal agent, reduces blood loss in a porcine model of trauma with dabigatran anticoagulation. J Am Coll Cardiol. 2015;66(13):1518-9.
50. Honickel M, Treutler S, van Ryn J, Tillmann S, Rossaint R, Grottke O. Reversal of dabigatran anticoagulation ex vivo: porcine study comparing prothrombin complex concentrates and idarucizumab. Thromb Haemost. 2015;113(4):728-40.

51. Glund S, Stangier J, Schmohl M, Gansser D, Norris S, van Ryn J, et al. Safety, tolerability, and efficacy of idarucizumab for the reversal of the anticoagulant effect of dabigatran in healthy male volunteers: a randomised, placebo-controlled, double-blind phase 1 trial. Lancet. 2015; 386(9994):680-90

52. Pollack Jr CV, Reilly PA, Eikelboom J, Glund S, Verhamme P, Bernstein RA, et al. Idarucizumab for dabigatran reversal. N Engl J Med. 2015;373(6):511-20.

53. Lindahl TL, Baghaei F, Blixter IF, Gustafsson KM, Stigendal L, Sten-Linder M, et al. Effects of the oral, direct thrombin inhibitor dabigatran on five common coagulation assays. Thromb Haemost. 2011;105(2):371-8. 